Im September 2007 startete in Leipzig ein dreijähriges Empowermentprojekt, das durch das Bundesprogramm „VIELFALT TUT GUT. Jugend für Vielfalt, Toleranz und Demokratie“ und den Freistaat Sachsen gefördert wird. Zielgruppe sind Jugendliche mit Migrationshintergrund und deren Eltern. Die drei Projektphasen sollen in ein Konzept für Empowermenttrainings unter spezifisch ostdeutschen Bedingungen für verschiedene Zielgruppen münden. In den ersten Trainings mit Jugendlichen aus einer Deutsch-als-Zweitsprache-Klasse und einer freien Gruppe von Jugendlichen mit unsicherem Aufenthalt geht es ums Kofferpacken: Ein leerer Koffer wird von den TeilnehmerInnen in einem sechsmonatigen Prozess langsam gefüllt, mit Ideen über das eigene Leben, Fähigkeiten und Ressourcen, aber auch mit selbst entwickelten Handlungsstrategien gegen blöde Anmachen, Ausgrenzung und Rassismus. Der Koffer steht symbolisch für den Rahmen, den wir TrainerInnen schaffen. Mit Methoden der Theater-, Körper-, Biographie- und Medienarbeit ermutigen wir die TeilnehmerInnen, ihre Fähigkeiten und Ressourcen zu erkennen und mit einem Griff in ihren selbst gepackten Ressourcenkoffer nutzbar zu machen. Die Jugendlichen, aber auch Eltern, rücken näher zusammen, hören einander zu und entdecken neue Möglichkeiten, trotz gesellschaftlicher Barrieren aktiv zu wer-

\title{
Empowerment durch Kofferpacken
} den. Das interkulturelle TrainerInnenteam steht diesen Prozes-

sen nicht im Weg, sondern schafft Platz und Bewusstsein für die selbst bestimmte Lebensführung, die das Team so schnell wie möglich wieder entbehrlich macht. Auf die spezifische Gruppe zugeschnitten wird ein Trainingsdesign aus den Modulen Selbststärkung, Antidiskriminierung und Selbstorganisation zusammengestellt und unter Einbeziehung des jeweils spezifischen institutionellen Umfeldes umgesetzt. Unser Empowermentansatz verbindet drei miteinander verwobene Ebenen im Umgang mit Rassismuserfahrungen. Auf der individuellen Ebene definiert er das Subjekt als handlungsfähiges und aktives Mitglied der Gesellschaft, das Rassismuserfahrungen nicht hilflos ausgeliefert ist. Auf gesellschaftlicher Ebene werden Beziehungen von Macht und Ohnmacht aufgedeckt und ungenügende Teilhabe an sozialem, ökonomischem und kulturellem Kapital bewusst gemacht. Rassismus wird als ein entpersonalisiertes Produkt gesellschaftlicher Prozesse greifbar. Auf sozialer Ebene werden soziale Netzwerke und Unterstützungspotentiale von Gleichgesinnten genutzt, um Möglichkeitsräume zu schaffen und gesellschaftliche Handlungsfähigkeit herzustellen. Die Trainings finden in drei Modulen statt: positive Identitätsentwicklung und Selbststärkung, Bewältigungsstrategien im Umgang mit Rassismus- und Diskriminierungserfahrungen und Stärkung von Selbstorganisation und Partizipation. Wo Empowermentprozesse beginnen und welche Erfolge sie haben, hängt sehr stark von den verschiedenen Gruppen und Einzelpersonen und ihren Lebenslagen ab. In einem sechsmonatigen Prozess lässt sich beobachten, dass durchaus kleine Schritte in Richtung positiver Identitätsentwicklung und Selbstverantwortung gemacht werden können. Gleichwohl muss darauf hingewiesen werden, dass Empowermentprozesse langwierige und schwere Entwicklungsprozesse beinhalten, die in einem halben Jahr nur angestoßen werden können. Das Umfeld muss die Prozesse begleiten und unterstützen. Isolierte Trainings, die nicht auf die Umgebung wirken und Veränderungen einfordern, sind wenig sinnvoll. Jugendliche, die sich ein neues Selbstbewusstsein erarbeiten, das von Stereotypen, Vorstellungen und Normierungen abweicht, laufen Gefahr, in einem nicht sensibilisierten Umfeld auf Ablehnung und Unverständnis zu treffen. In diesem Sinne ist die Empowermentarbeit auf alle Akteure wie LehrerInnen und Familienmitglieder auszuweiten. Wenn solch ein Umfeld geschaffen ist, ist es an den TrainerInnen, ihre Nutzlosigkeit zu erkennen. 